\title{
Layer dependence of stacking order in nonencapsulated few-layer $\mathrm{Crl}_{3}$
}

\author{
Kai Guo ${ }^{1,4}$, Bowen Deng ${ }^{3}$, Zhen Liu ${ }^{1,4}$, Chaofeng Gao ${ }^{2}$, Zhongtai Shi ${ }^{1,4}$, Lei Bi ${ }^{1,4}$, Li Zhang ${ }^{1,4}$, \\ Haipeng Lu ${ }^{1,4}$, Peiheng Zhou ${ }^{1,4}$, Linbo Zhang ${ }^{1,4^{*}}$, Yingchun Cheng ${ }^{2}$ and Bo Peng ${ }^{1,4^{*}}$
}

\begin{abstract}
Long-range magnetic orders in atomically thin ferromagnetic $\mathrm{CrI}_{3}$ trigger new fascinating physics and application perspectives. The physical properties of two-dimensional (2D) ferromagnetism $\mathrm{CrI}_{3}$ are significantly influenced by interlayer spacing and stacking order, which are sensitive to the hydrostatic pressure and external environments. However, there remains debate on the stacking order at low temperature. Here, we study the interlayer coupling and stacking order of non-encapsulated 2-5 layer and bulk $\mathrm{CrI}_{3}$ at $10 \mathrm{~K}$ by Raman spectroscopy; demonstrate a rhombohedral stacking in both antiferromagnetic and ferromagnetic $\mathrm{CrI}_{3}$. The opposite helicity dependence of $A_{g}$ and $E_{g}$ modes arising from phonon symmetry further validates the rhombohedral stacking. An anomalous temperature-dependent behavior is observed due to spin-phonon coupling below $60 \mathrm{~K}$. Our study provides insights into the interlayer coupling and stacking orders of $2 \mathrm{D}$ ferromagnetic materials.
\end{abstract}

Keywords: ferromagnetic 2D materials, Ising ferromagnet, spintronics, magneto-optical

\section{INTRODUCTION}

Since the discovery of two ferromagnetic atomically thin $\mathrm{CrI}_{3}$ and $\mathrm{Cr}_{2} \mathrm{Ge}_{2} \mathrm{Te}_{6}$ in 2017, intrinsic ferromagnetism in two-dimensional (2D) van der Waals (vdW) materials, maintaining long-range magnetic orders at the atomic monolayer limit, has received increasing attention [1-7]. $2 \mathrm{D} v d W$ ferromagnetic materials as spin-filters have been integrated into vdW heterostructures, demonstrating giant tunneling magnetoresistance towards the nextgeneration information transfer and data storage technologies [8-10]. Among 2D ferromagnetic materials, $\mathrm{CrI}_{3}$ is of particular interest. Bulk $\mathrm{CrI}_{3}$ is ferromagnetic below the Curie temperature $\left(T_{\mathrm{C}}\right)$, but few-layer $\mathrm{CrI}_{3}$ displays striking layer-dependent magnetism. Each individual layer is ferromagnetic; however, adjacent layers are antiferromagnetically coupled together and could become a layered antiferromagnet when thinned down to a few atomic layers [1]. The interlayer magnetic state can be switched between ferromagnetic and antiferromagnetic by electric gating or electrostatic doping [11-14] and pressure [15,16].

In a vdW material and heterostructures, a tiny change of lattice constant and interlayer coupling between adjacent layers can drastically influence their physical properties. Bulk $\mathrm{CrI}_{3}$ adopts the monoclinic stacking at room temperature, while transfers to rhombohedral stacking at $\sim 210 \mathrm{~K}$. Bulk and encapsulated few-layer $\mathrm{CrI}_{3}$ with rhombohedral stacking have been reported to be ferromagnetic below $\sim 61 \mathrm{~K}[17,18]$. However, recent experiments demonstrate that the $\mathrm{BN}$-encapsulated bi- and few-layer $\mathrm{CrI}_{3}$ and $\mathrm{CrCl}_{3}$ belong to monoclinic structure (point group $C_{2 h}(2 / m)$ ) rather than rhombohedral structure at low temperature $[19,20]$, and some theoretical proposals demonstrate that the antiferromagnetic coupling is associated with monoclinic layer stacking [21]. Thus, there is still a debate on the stacking order of $\mathrm{CrI}_{3}$ at low temperature.

\footnotetext{
${ }^{1}$ National Engineering Research Center of Electromagnetic Radiation Control Materials, School of Electronic Science and Engineering, University of Electronic Science and Technology of China, Chengdu 611731, China

${ }^{2}$ Key Laboratory of Flexible Electronics \& Institute of Advanced Materials, Jiangsu National Synergetic Innovation Center for Advanced Materials, Nanjing Tech University, Nanjing 211816, China

${ }^{3}$ Department of Physics, Broida Hall, University of California Santa Barbara, CA 93106-9530, USA

${ }^{4}$ Key Laboratory of Multi-spectral Absorbing Materials and Structures of Ministry of Education, University of Electronic Science and Technology of China, Chengdu 611731, China

* Corresponding authors (emails: bo_peng@uestc.edu.cn (Peng B); zhanglinbo@uestc.edu.cn (Zhang L))
} 
A complete understanding of the low-temperature lattice structure and stacking order is crucial for $2 \mathrm{D} v \mathrm{vW}$ ferromagnetic materials. Raman spectroscopy is a powerful tool to study the crystal structures, lattice vibration and shift and interlayer coupling [22-28]. In this study, we investigate the layer, polarization and helicity dependencies of Raman features of non-encapsulated 2-5 layer and bulk $\mathrm{CrI}_{3}$ at $10 \mathrm{~K}$, demonstrating that few-layer and bulk $\mathrm{CrI}_{3}$ are rhombohedral phase at low temperature, including antiferromagnetic bilayer $\mathrm{CrI}_{3}$. The peak intensities of $\mathrm{E}_{\mathrm{g}}^{3}$ and $\mathrm{E}_{\mathrm{g}}^{4}$ mode are independent of the polarization angle, while the intensities of $\mathrm{A}_{\mathrm{g}}^{2}$ and $\mathrm{A}_{\mathrm{g}}^{3}$ show a two-fold polarization dependence. The polarization-resolved Raman spectra demonstrate that the $\mathrm{A}_{\mathrm{g}}^{3}$ and $\mathrm{E}_{\mathrm{g}}^{4}$ features show opposite helicities in which the $\mathrm{A}_{\mathrm{g}}^{3}$ mode maintains the helicity of incident light; however, the $\mathrm{E}_{\mathrm{g}}^{4}$ mode reverses it. Under cooling, the $\mathrm{A}_{\mathrm{g}}^{3}$ and $\mathrm{E}_{\mathrm{g}}^{4}$ modes shift to higher frequencies; remarkably, a spinphonon coupling takes place and causes an anomalous behavior for the Raman feature below $\sim 60 \mathrm{~K}$.

\section{EXPERIMENTAL METHODS}

\section{Sample preparation}

Few-layer $\mathrm{CrI}_{3}$ was mechanically exfoliated from a bulk crystal onto polydimethylsiloxane films and was then directly transferred onto $\mathrm{SiO}_{2} / \mathrm{Si}$ substrates, which was then loaded into the cold head for optical measurements in a glove box filled with Ar.

\section{Optical measurements}

The Raman signals were recorded using a Witec Alpha 300R Plus confocal Raman microscope with a closed cycle optical cryostat $(10 \mathrm{~K})$ and a $7 \mathrm{~T}$ magnetic field. A powerstabilized $633 \mathrm{~nm}$ HeNe laser was modulated by photoelastic modulator (PEM) and coupled to the Witec Raman system. The modulated beam was directed through a non-polarizing beamsplitter cubes to the sample, housed in a closed-cycle cryostat at $10 \mathrm{~K}$. An out-of-plane magnetic field was applied in Faraday geometry. The reflected beam passed through the same non-polarizing beamsplitter cubes, non-PM multimode fiber onto a photodetector, where lock-in detection measured the reflected intensity at $f_{\mathrm{PEM}}(50 \mathrm{kHz})$. A long working distance $50 \times$ objective (NA = 0.45) was used for the Raman and reflective magnetic circular dichroism (RMCD) measurements. The Raman signals were coupled into the spectrometer with an 1800 and $600 \mathrm{~g} \mathrm{~mm}^{-1}$ grating. $1 / 4 \lambda$ waveplate and polarization analyzer were used for the polarization-resolved Raman measurements. The power of a $514 \mathrm{~nm}$ laser was measured to be approximately $2 \mathrm{~mW}$, and the typical integration time was $30 \mathrm{~s}$.

\section{RESULTS AND DISCUSSION}

\section{Layer dependence of magnetic order}

In 2D ferromagnetic $\mathrm{CrI}_{3}$, the $\mathrm{Cr}^{3+}$ ions in each layer are coordinated by six nonmagnetic $\mathrm{I}^{-}$ions to form an octahedral geometry, which further share edges to build a honeycomb network (Fig. 1a). Bulk $\mathrm{CrI}_{3}$ crystals undergo a phase transition to a rhombohedral structure (space group $R \overline{3}$, Fig. $1 b)$ at $\sim 210-220 \mathrm{~K}$ from a monoclinic structure (space group $\mathrm{C} 2 / \mathrm{m}$, Fig. 1c) at room temperature [17]. Fig. 1d shows the non-encapsulated $\mathrm{CrI}_{3}$ with 2-6 layers on $\mathrm{SiO}_{2}(300 \mathrm{~nm}) / \mathrm{Si}$ substrates, which are insitu loaded into cold head with an optical window in the glovebox. The different numbers of layers can be identified through the optical contrast. Our experimental optical contrast results shown in Fig. 1e (red circles) are consistent with the reported experimental and calculated results from Xu et al. [1] (solid blue line in Fig. 1e). Bulk $\mathrm{CrI}_{3}$ crystals show distinct ferromagnetism below $\sim 65 \mathrm{~K}$ (Fig. 1f).

Fig. 2 shows the RMCD signal in atomically-thin $\mathrm{CrI}_{3}$ at $10 \mathrm{~K}$. RMCD signals from a bilayer $\mathrm{CrI}_{3}$ flake approach zero when applied magnetic fields $\pm 0.65 \mathrm{~T}$, indicating antiferromagnetic behavior (Fig. 2a). In bilayer $\mathrm{CrI}_{3}$, the two layers have opposite magnetic orientation and nearly compensate for the intrinsic magnetocrystalline anisotropy each other; therefore, the net magnetization disappear and 2-layer (2L) $\mathrm{CrI}_{3}$ is antiferromagnetic [1,2]. However, striking hysteresis is observed in $3 \mathrm{~L}$ and $4 \mathrm{~L} \mathrm{CrI}_{3}$ flakes (Fig. 2b, c). In $3 \mathrm{~L} \mathrm{CrI}_{3}$, the magnetizations of the three layers are oriented to the same direction, while the magnetization of one layer is opposite with the other three in $4 \mathrm{~L} \mathrm{CrI}_{3}$. Thus, the net magnetization of $3 \mathrm{~L} \mathrm{CrI}_{3}$ is stronger than that of $4 \mathrm{~L} \mathrm{CrI}_{3}$.

\section{Layer dependence of stacking order}

The stacking order and crystal structures of nonencapsulated few-layer $\mathrm{CrI}_{3}$ can be identified by polarized Raman spectroscopy. The polarization selection rules were performed on a $2-5$ layer $(2 \mathrm{~L}, 3 \mathrm{~L}, 4 \mathrm{~L}$ and $5 \mathrm{~L}$ ) and bulk $\mathrm{CrI}_{3}$ at $10 \mathrm{~K}$ using a confocal Raman microscope system with a backscattering geometry (Fig. 3a-d). The incident and scattered light are along the $-Z$ and $+Z$ direction, respectively. The polarization configuration of 
a

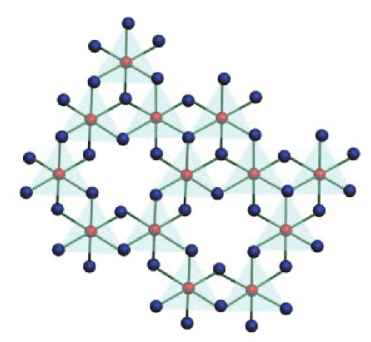

d

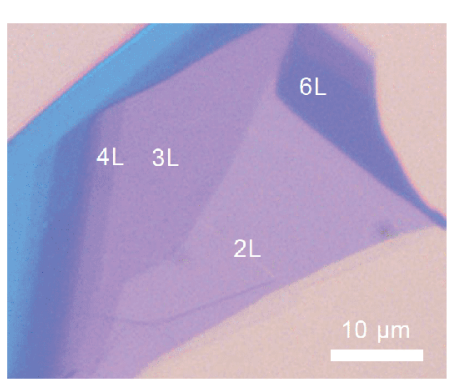

b

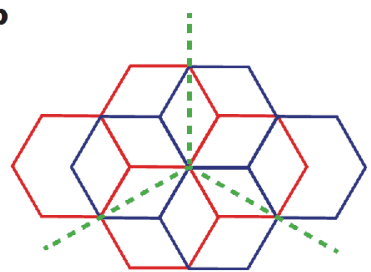

S6 $R \overline{3}$

Rhombohedral

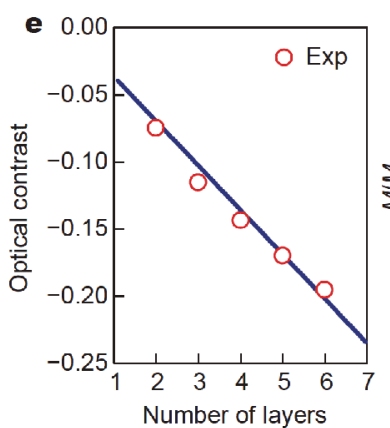

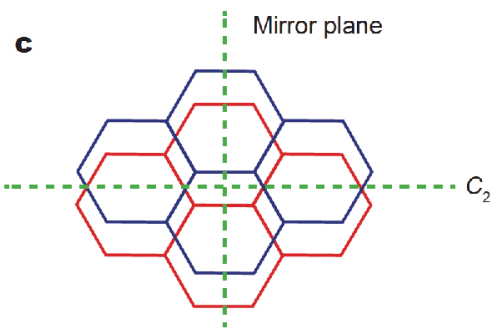

$\mathrm{C}_{2 h} \quad \mathrm{C} 2 / \mathrm{m}$

Monoclinic

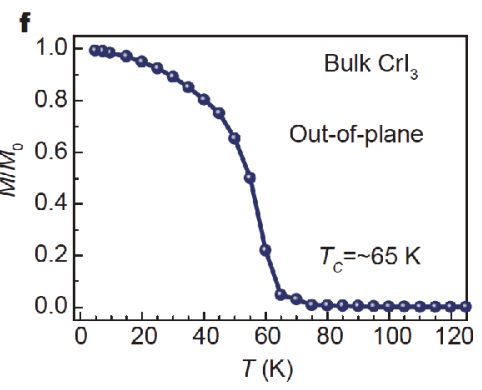

Figure 1 Exfoliated few-layer $\mathrm{CrI}_{3}$. (a) Atomic structures of monolayer $\mathrm{CrI}_{3}$. (b, c) Rhombohedral (b) and monoclinic (c) stacking order in bilayer $\mathrm{CrI}_{3}$. The rhombohedral structure has an out-of-plane $C_{3}$ axis and a symmetric center $\left(S_{6}=C_{3}+i\right)$, while the monoclinic structure has an in-plane $C_{2}$ axis and a mirror plane. (d) Optical micrograph of the exfoliated few-layer $\mathrm{CrI}_{3}$. (e) Optical contrast of the CrI $\mathrm{C}_{3}$ samples with different numbers of layers (red circles). The blue solid line is the calculated results based on Fresnel's equations [1]. (f) Magnetization of bulk CrI $\mathrm{f}_{3}$ as a function of temperature, indicating $T_{\mathrm{C}}=\sim 65 \mathrm{~K}$.
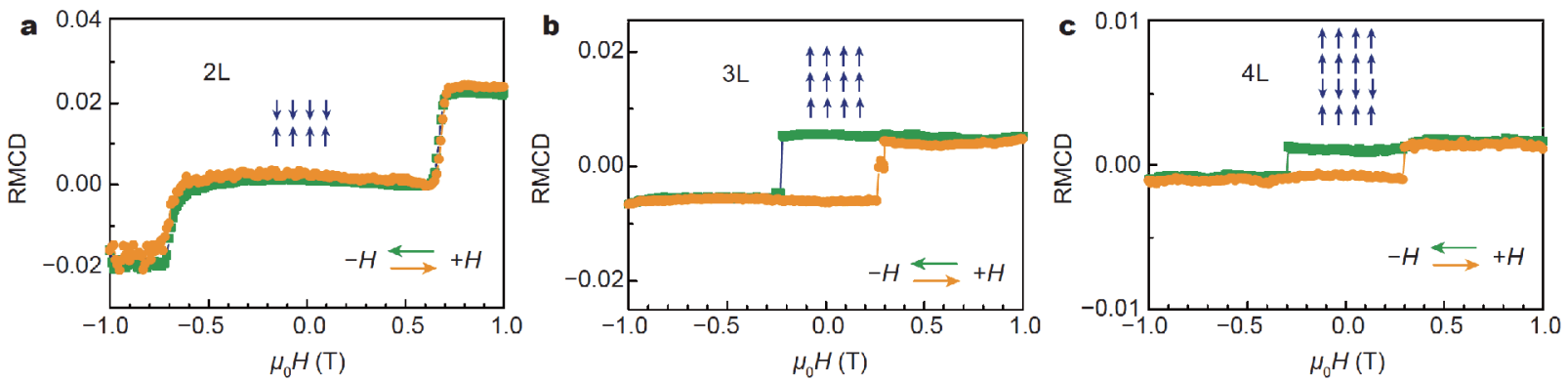

Figure 2 Layer-dependent magnetic ordering in atomically-thin $\mathrm{CrI}_{3}$ at $10 \mathrm{~K}$. RMCD signal on a $2 \mathrm{~L}$ (a), 3L (b) and $4 \mathrm{~L}$ (c) $\mathrm{CrI} \mathrm{I}_{3}$ flake, showing antiferromagnetic behavior in bilayer $\mathrm{CrI}_{3}$ and ferromagnetic behavior in $3 \mathrm{~L}$ and $4 \mathrm{~L} \mathrm{CrI}_{3}$. The blue arrows indicate the magnetization orientation in different layers.

the incident and scattered light are parallel $(X X)$ or perpendicular $(X Y)$ to each other. The feature at $\sim 103$ and $128 \mathrm{~cm}^{-1}$ is only present in the parallel configuration $(X X)$, while the Raman peaks at $\sim 107$ and $\sim 238 \mathrm{~cm}^{-1}$ appear for both parallel and perpendicular configurations $(X X$ and $X Y)$. The Raman scattering intensities $I$ are proportional to $\left|\boldsymbol{e}_{\mathrm{s}} \cdot \boldsymbol{R} \cdot \boldsymbol{e}_{\mathrm{i}}\right|^{2}$, where $\boldsymbol{e}_{\mathrm{i}}$ and $\boldsymbol{e}_{\mathrm{s}}$ are the polarization unit vectors of the incident and scattered light, respectively $[26,29,30] . \boldsymbol{R}$ is the Raman tensor of the Raman-active vibrational modes as predicted by group theory.

We consider both the rhombohedral (point group, $C_{3 i}$ ) and monoclinic phase (point group, $C_{2 h}$ ). The scattered light polarization has an angle of $\theta$ with that of the incident light, which is parallel to the $x$-axis. Thus, $\widehat{\boldsymbol{e}_{\mathrm{i}}}=(1,0,0)$ and $\widehat{\boldsymbol{e}}_{\mathrm{s}}=(\cos \theta, \sin \theta, 0)$, and $\theta=0^{\circ}$ and $90^{\circ}$ under the $X X$ and $X Y$ polarization configuration.

In rhombohedral structure, Raman active modes are the $\mathrm{A}_{\mathrm{g}}$ and $\mathrm{E}_{\mathrm{g}}$ modes and the corresponding Raman tensors are given by [31] 

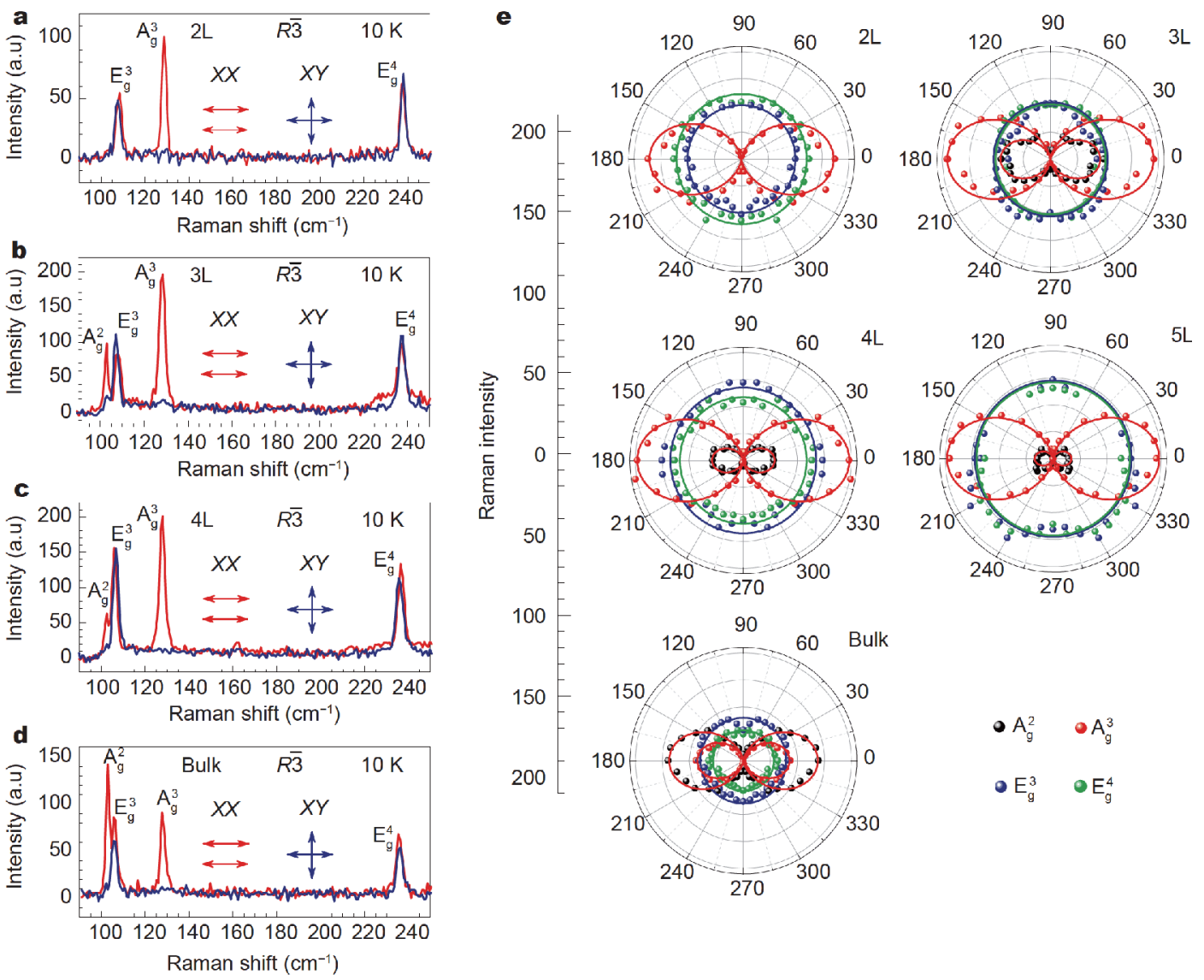

Figure 3 Layer dependence of the stacking orders in $\mathrm{CrI}_{3}$ at $10 \mathrm{~K}$. Raman spectra of $2 \mathrm{~L}$ (a), $3 \mathrm{~L}$ (b), $4 \mathrm{~L}$ (c) and bulk CrI 3 (d) in the parallel (XX, red curve) and perpendicular (XY, blue curve) polarization selection channels. (e) Polarization angle dependence of Raman intensity in $2-5$ layer and bulk $\mathrm{CrI}_{3}$. The Raman features at $\sim 107$ and $\sim 234 \mathrm{~cm}^{-1}$ are degenerated $\mathrm{E}_{\mathrm{g}}^{3}$ and $\mathrm{E}_{\mathrm{g}}^{4}$ mode for the Rhombohedral phase, whose intensities are independent of the polarization angle; in contrast, the $\mathrm{A}_{\mathrm{g}}^{2}$ and $\mathrm{A}_{\mathrm{g}}^{3}$ modes at $\sim 103$ and $128 \mathrm{~cm}^{-1}$ show distinct a two-fold polarization dependence.

$$
\begin{aligned}
& \mathrm{A}_{\mathrm{g}}(\boldsymbol{R}):\left(\begin{array}{ccc}
a & 0 & 0 \\
0 & a & 0 \\
0 & 0 & b
\end{array}\right),{ }^{1} \mathrm{E}_{\mathrm{g}}(\boldsymbol{R}):\left(\begin{array}{ccc}
c & d & e \\
d & -c & f \\
e & f & 0
\end{array}\right), \\
& { }^{2} \mathrm{E}_{\mathrm{g}}(\boldsymbol{R}):\left(\begin{array}{ccc}
d & -c & -f \\
-c & -d & e \\
-f & e & 0
\end{array}\right) .
\end{aligned}
$$

Taking $\boldsymbol{e}_{\mathrm{i}}, \boldsymbol{e}_{\mathrm{s}}$ and $\boldsymbol{R}$ to $I \propto\left|\boldsymbol{e}_{\mathrm{s}} \cdot \boldsymbol{R} \cdot \boldsymbol{e}_{\mathrm{i}}\right|^{2}$, the Raman intensities of $\mathrm{A}_{\mathrm{g}}$ and $\mathrm{E}_{\mathrm{g}}$ are

$I_{\mathrm{A}_{\mathrm{g}}}(\boldsymbol{R}) \propto a^{2} \cos ^{2} \theta$,

${ }^{1} I_{\mathrm{E}_{\mathrm{g}}}(\boldsymbol{R}) \propto c^{2} \cos ^{2} \theta+c d \sin (2 \theta)+d^{2} \sin ^{2} \theta$,

${ }^{2} I_{\mathrm{E}_{\mathrm{g}}}(\boldsymbol{R}) \propto d^{2} \cos ^{2} \theta-c d \sin (2 \theta)+c^{2} \sin ^{2} \theta$.

The $\mathrm{E}_{\mathrm{g}}$ modes are degenerated. All $\mathrm{E}_{\mathrm{g}}$ modes contribute to a peak at the same frequency, so the Raman intensities of $E_{\mathrm{g}}$ modes can be expressed as

$$
I_{\mathrm{E}_{\mathrm{g}}}(\boldsymbol{R})={ }^{1} I_{\mathrm{E}_{\mathrm{g}}}(\boldsymbol{R})+{ }^{2} I_{\mathrm{E}_{\mathrm{g}}}(\boldsymbol{R})=c^{2}+d^{2},
$$

as a constant, and the $\mathrm{E}_{\mathrm{g}}$ modes are independent of the polarization, which can be detected in any polarization configuration. In contrast, the $\mathrm{A}_{\mathrm{g}}$ modes show striking two-fold polarization dependence, which can be observed only in parallel polarization configuration.

In monoclinic structure, the symmetry is lowered and the $E_{g}$ modes split to an $A_{g}$ and a $B_{g}$ mode. Thus, only the $\mathrm{A}_{\mathrm{g}}$ and $\mathrm{B}_{\mathrm{g}}$ modes are active and corresponding Raman tensors are given by

$$
\mathrm{A}_{\mathrm{g}}(\boldsymbol{M}):\left(\begin{array}{lll}
a & 0 & d \\
0 & b & 0 \\
d & 0 & c
\end{array}\right), \mathrm{B}_{\mathrm{g}}(\boldsymbol{M}):\left(\begin{array}{lll}
0 & e & 0 \\
e & 0 & f \\
0 & f & 0
\end{array}\right) \text {. }
$$

The Raman intensities of $\mathrm{A}_{\mathrm{g}}$ and $\mathrm{B}_{\mathrm{g}}$ can be expressed as 


$$
I_{\mathrm{A}_{\mathrm{g}}}(\boldsymbol{M}) \propto a^{2} \cos ^{2} \theta, I_{\mathrm{Bg}_{\mathrm{g}}}(\boldsymbol{M}) \propto e^{2} \sin ^{2} \theta .
$$

Thus, the Raman intensities of $\mathrm{A}_{\mathrm{g}}$ and $\mathrm{B}_{\mathrm{g}}$ modes have opposite two-fold dependence on polarization angle in the monoclinic phase.

Therefore, group theory predicts that the Raman scattering intensity is zero for $\mathrm{A}_{\mathrm{g}}$ modes under the perpendicular $(X Y)$ configuration in both the rhombohedral and monoclinic stacking [30]. However, only for rhombohedral phase, there remain the polarization-independent $\mathrm{E}_{\mathrm{g}}$ modes that have nonzero Raman intensities under the parallel $(X X)$ and perpendicular $(X Y)$ configurations. The polarization angle dependences of the features at $\sim 103$, $\sim 107, \sim 128$ and $\sim 234 \mathrm{~cm}^{-1}$ of non-encapsulated 2-5 layer and bulk $\mathrm{CrI}_{3}$ are shown in Fig. 3e. The Raman intensities of the peaks near 103 and $128 \mathrm{~cm}^{-1}$ have a two-fold pattern versus polarization angle; in contrast, the modes at $\sim 107$ and $\sim 234 \mathrm{~cm}^{-1}$ are independent of the polarization angle. Therefore, the non-encapsulated 2-5 layer and bulk $\mathrm{CrI}_{3}$ are rhombohedral phase rather than monoclinic phase; and the peaks at $\sim 103, \sim 107, \sim 128$ and $\sim 234 \mathrm{~cm}^{-1}$ are assigned to the $\mathrm{A}_{\mathrm{g}}^{2}, \mathrm{E}_{\mathrm{g}}^{3}, \mathrm{~A}_{\mathrm{g}}^{3}$ and $\mathrm{E}_{\mathrm{g}}^{4}$ modes, respectively
[18].

To further validate the rhombohedral stacking of $\mathrm{CrI}_{3}$ at low temperature, the helicity selection rules were studied at $10 \mathrm{~K}$ (Fig. 4). Fig. 4a shows a schematic of the experimental optical setup. The linearly polarized light passes through a $1 / 4 \lambda$ waveplate and transfers to circularly polarized incident $e_{\mathrm{i}}=E_{0} e^{i(k z-w t)}(\widehat{x} \pm i \widehat{y})$. The incident and scattered light are along opposite direction, and thus, the scattered light $e_{\mathrm{s}}$ is $E_{0} e^{i(k z-w t)}(\widehat{x} \mp i \widehat{y})$ and $E_{0} e^{i(k z-w t)}(\widehat{x} \pm i \widehat{y})$ under the same $(\sigma+\sigma+)$ and opposite $\left(\sigma+\sigma^{-}\right)$circular polarization configuration, respectively.

The Raman intensities of the $\mathrm{A}_{\mathrm{g}}$ modes in rhombohedral phase can be expressed as

$I_{\mathrm{A}_{\mathrm{g}}}^{\sigma^{+\sigma+}}(\boldsymbol{R}) \propto a^{2}, I_{\mathrm{Ag}_{\mathrm{g}}}^{\sigma+\sigma^{-}}(\boldsymbol{R}) \propto 0$.

On the contrary, the Raman intensities of the $\mathrm{E}_{\mathrm{g}}$ modes can be expressed as

$I_{\mathrm{E}_{\mathrm{g}}}^{\sigma+\sigma^{+}}(\boldsymbol{R}) \propto 0, I_{\mathrm{E}_{\mathrm{g}}}^{\sigma+\sigma^{-}}(\boldsymbol{R}) \propto c^{2}+d^{2}$.

As shown in Equations $(6,7)$, the $\mathrm{A}_{\mathrm{g}}$ modes maintain the helicity of incident light; however, the $\mathrm{E}_{\mathrm{g}}$ modes re-
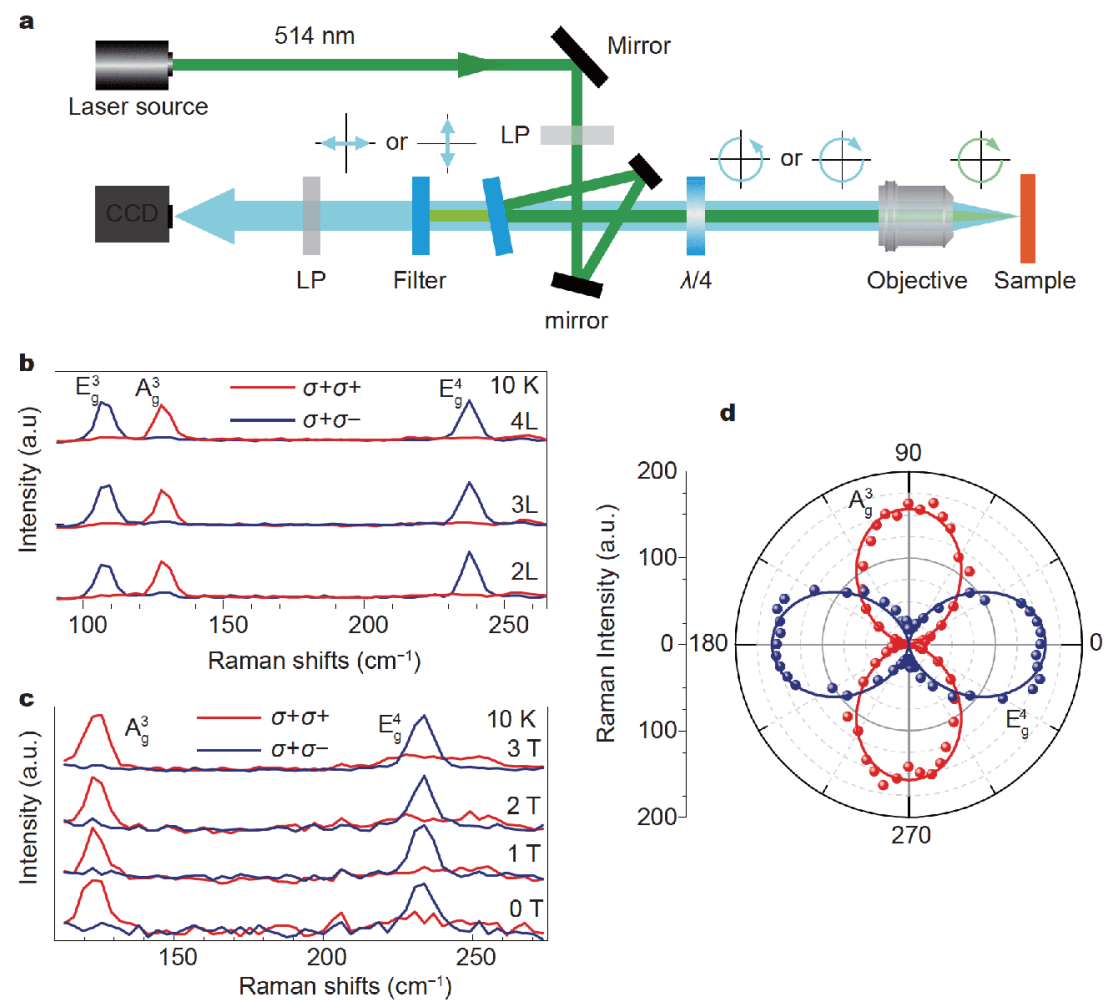

Figure 4 Helicity-resolved Raman spectra at $10 \mathrm{~K}$. (a) Schematic of the helicity-resolved experimental optical setup. (b) Helicity-resolved Raman spectra of $2 \mathrm{~L}, 3 \mathrm{~L}$ and $4 \mathrm{~L} \mathrm{CrI}_{3}$. (c) Helicity-resolved Raman spectra of $3 \mathrm{~L} \mathrm{CrI}_{3}$ as a function of the magnetic field. (d) Corresponding angular dependence of the $\mathrm{A}_{\mathrm{g}}^{3}$ and $\mathrm{E}_{\mathrm{g}}^{4}$ mode intensities for $3 \mathrm{~L} \mathrm{CrI}_{3}$, showing a distinctly opposite helicity. 
verse it. In contrast, for monoclinic phase with lower symmetry, both the $A_{g}$ and $B_{g}$ modes reverse the helicity of incident light, in which the Raman intensities of $A_{g}$ and $\mathrm{B}_{\mathrm{g}}$ modes are given by

$$
\begin{aligned}
& I_{\mathrm{A}_{\mathrm{g}}}^{\sigma+\sigma^{+}}(\boldsymbol{M}) \propto 0, I_{\mathrm{A}_{\mathrm{g}}}^{\sigma+\sigma^{-}}(\boldsymbol{M}) \propto a^{2}, \\
& I_{\mathrm{B}_{\mathrm{g}}}^{\sigma+\sigma^{+}}(\boldsymbol{M}) \propto 0, I_{\mathrm{B}_{\mathrm{g}}}^{\sigma+\sigma^{-}}(\boldsymbol{M}) \propto e^{2} .
\end{aligned}
$$

The polarization-resolved Raman spectra were obtained under excitation by a left-handed circularly polarized light at $2.41 \mathrm{eV}(\sigma+)$. Remarkably, the Raman features of $2 \mathrm{~L}, 3 \mathrm{~L}$ and $4 \mathrm{~L} \mathrm{CrI}_{3}$ at $\sim 128 \mathrm{~cm}^{-1}$ have the same helicity as the incident photon, whereas the peaks at $\sim 234 \mathrm{~cm}^{-1}$ switch the helicity (Fig. $4 \mathrm{~b}$ and c). The striking opposite polarization dependence of helicities of the two Raman modes is shown in Fig. 4 d. The experimental data are in agreement with the predicted rhombohedral phase results. The helicity-dependent experimental results further validate that the peaks at $\sim 128$ and $\sim 234 \mathrm{~cm}^{-1}$ are assigned to the $\mathrm{A}_{\mathrm{g}}^{3}$ and $\mathrm{E}_{\mathrm{g}}^{4}$ modes, respectively, consistent with above polarization-resolved Raman results. With increasing magnetic field, the helicity selection behaviors show no detectable changes (Fig. 4c), indicating that this is independent of the external magnetic field. The helicity selection behaviors are only attributed to the symmetric Raman tensors. Thus, 2-5 layer and bulk 2D ferromagnetic $\mathrm{CrI}_{3}$ have rhombohedral stacking order.

\section{Temperature dependence of the Raman features}

To better understand the lattice dynamics, the temperature dependence of the Raman features was determined in $2 \mathrm{~L}, 3 \mathrm{~L}$ and $6 \mathrm{~L} \mathrm{CrI}_{3}$ flakes. Since the transition from the monoclinic phase to the rhombohedral phase occurs at around 210-220 K in $\mathrm{CrI}_{3}$ [17], we focus on investigating the Raman features below $200 \mathrm{~K}$. Fig. $5 \mathrm{a}-\mathrm{c}$ show the normalized Raman intensity maps of the $2 \mathrm{~L}, 3 \mathrm{~L}$ and $6 \mathrm{~L} \mathrm{CrI}_{3}$ with decreasing temperatures from 200 to $10 \mathrm{~K}$. The $\mathrm{A}_{\mathrm{g}}^{3}$ and $\mathrm{E}_{\mathrm{g}}^{4}$ modes simultaneously blueshift to higher frequencies with decreasing temperatures, which are attributed to the phonon anharmonic decay and lattice contraction. The shifts of the $\mathrm{A}_{\mathrm{g}}^{3}$ modes are $\sim 1.4, \sim 0.9$ and $\sim 0.8 \mathrm{~cm}^{-1}$ for the $2 \mathrm{~L}, 3 \mathrm{~L}$ and $6 \mathrm{~L} \mathrm{CrI}_{3}$, and the $\mathrm{E}_{\mathrm{g}}^{4}$ modes show shifts of $\sim 2.9, \sim 2.9$ and $\sim 2.4 \mathrm{~cm}^{-1}$, respectively.
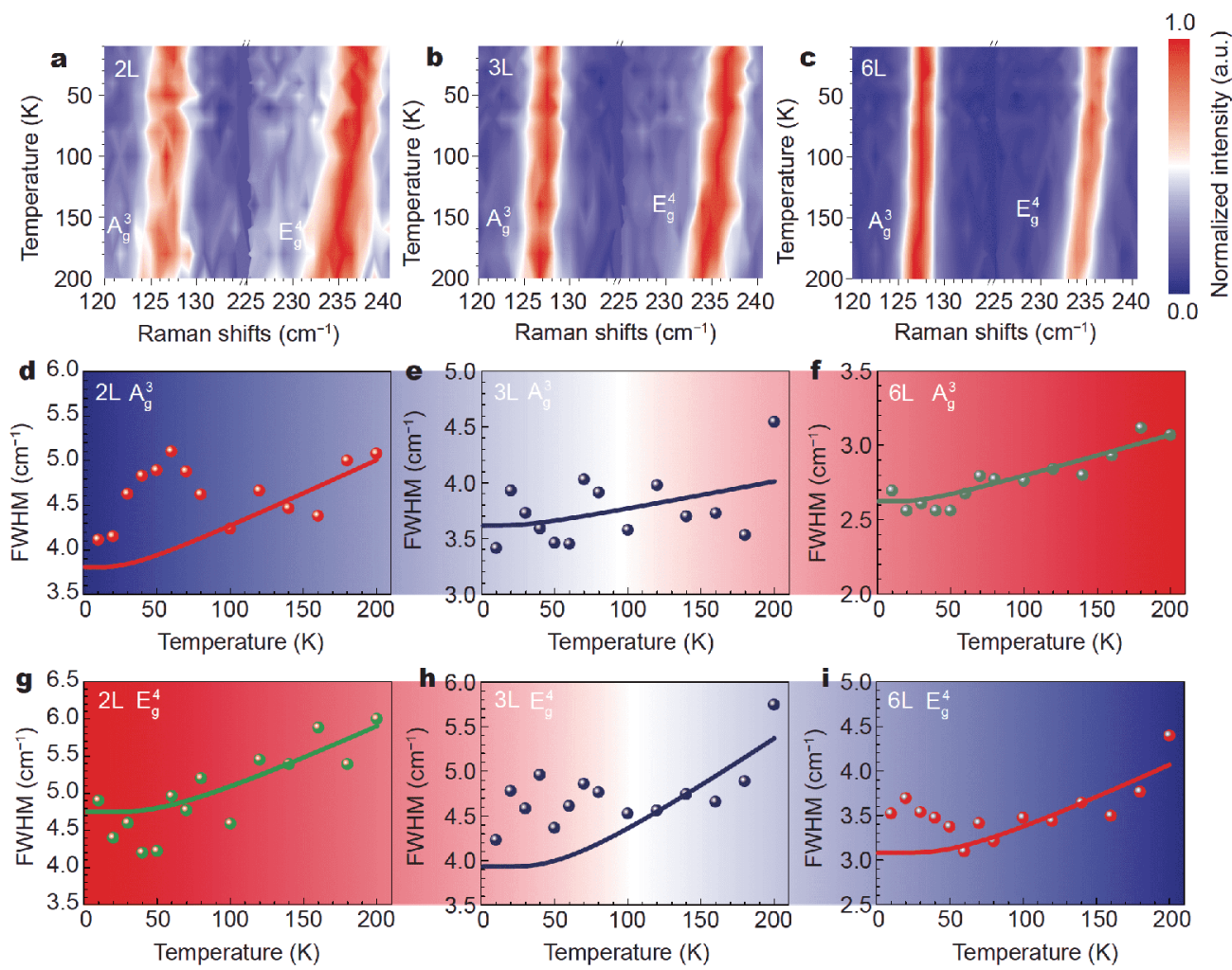

Figure 5 Temperature dependence of the $\mathrm{A}_{\mathrm{g}}^{3}$ and $\mathrm{E}_{\mathrm{g}}^{4}$ Raman modes. $(\mathrm{a}-\mathrm{c})$ Normalized Raman intensity maps of the $2 \mathrm{~L}, 3 \mathrm{~L}$ and $6 \mathrm{~L}$ CrI ${ }_{3}$ as a function of temperature. The Raman features shift to higher frequencies with decreasing temperatures. (d-i) The linewidths of the $\mathrm{A}_{\mathrm{g}}^{3}$ and $\mathrm{E}_{\mathrm{g}}^{4}$ Raman features as a function of temperature as extracted from Fig. 3a, b. The sudden increases in the linewidths indicate the occurrence of spin-phonon coupling. 
The linewidths for the $\mathrm{A}_{\mathrm{g}}^{3}$ and $\mathrm{E}_{\mathrm{g}}^{4}$ modes are extracted to investigate the spin-phonon coupling behavior. Fig. $5 \mathrm{~d}-\mathrm{i}$ show the linewidths of the $\mathrm{A}_{\mathrm{g}}^{3}$ and $\mathrm{E}_{\mathrm{g}}^{4}$ modes of $2 \mathrm{~L}, 3 \mathrm{~L}$ and $6 \mathrm{~L} \mathrm{CrI}_{3}$ with decreasing temperature from 200 to $10 \mathrm{~K}$. The temperature dependence of the linewidths is predicted by phonon-phonon coupling [32], which is given by

$\Gamma_{\mathrm{L}}(T)=\Gamma_{\mathrm{L}, 0}\left(1+2 \lambda_{\mathrm{ph}-\mathrm{ph}} /\left(\exp \left(h C \omega_{0} / 2 k_{\mathrm{B}} T\right)-1\right)\right)$,

where the $\Gamma_{\mathrm{L}, 0}$ and $\omega_{0}$ are the zero temperature limits of the linewidth and phonon energy, obtained by extrapolating the experimental results to $0 \mathrm{~K}$. The $\lambda_{\text {ph-ph }}$ represents the phonon-phonon coupling constant. Above $60 \mathrm{~K}\left(\sim T_{\mathrm{C}}\right)$, the linewidths of the $\mathrm{A}_{\mathrm{g}}^{3}$ and $\mathrm{E}_{\mathrm{g}}^{4}$ modes monotonically decrease as the temperature decreases (solid lines in Fig. 5d-i). However, the linewidths deviate from the expected tendency below $60 \mathrm{~K}$. In particular, the $\mathrm{A}_{\mathrm{g}}^{3}\left(\mathrm{E}_{\mathrm{g}}^{4}\right)$ modes of the $2 \mathrm{~L}(6 \mathrm{~L}) \mathrm{CrI}_{3}$ exhibit distinct deviation behavior (Fig. $5 \mathrm{~d}-\mathrm{i}$ ), which indicates that a new scattering mechanism contributes to the anomalous phonon behavior. The $T_{\mathrm{C}}$ of few-layer $\mathrm{CrI}_{3}$ is $\sim 65 \mathrm{~K}$ (Fig. 1f) [3]. The emerging long-range magnetic order is anticipated to result in the abrupt change in the linewidth originating from spin-phonon coupling. We briefly discuss the mechanism of spin-phonon coupling. The total Hamiltonian of lattice in crystals is expressed as

$H=H_{\text {ion }}+H_{\text {electron }}+H_{\text {e-ph }}$.

In the presence of magnetic orders, an additional Hamiltonian term is given by

$H_{\text {spin }}=-\sum_{i, j} J_{i j}\left\langle S_{i} S_{j}\right\rangle$,

where $J_{i j}$ is the spin-phonon coupling constant and $\left\langle S_{i} S_{j}\right\rangle$ is spin correlation function [33]. In $2 \mathrm{D}$ layered $\mathrm{CrI}_{3}$ materials, the electron-phonon coupling was not observed, thus the Hamiltonian $H_{\mathrm{e}-\mathrm{ph}}$ was neglected and only phonon-phonon coupling was considered. Thus, the total potential energy of a Raman active mode consists of "lattice" and "spin" contributions; corresponding $\mathrm{Ha}$ miltonian of Raman modes is expressed as

$H=H_{\text {ion }}+H_{\text {electron }}+H_{\text {spin }}=H_{\text {lattice }}+H_{\text {spin }}$.

Therefore, the spin-phonon exchange interaction takes place in the ferromagnetic phase at low temperature, resulting in the deviation of linewidth.

\section{CONCLUSION}

In summary, we demonstrated a comprehensive under- standing of stacking order of $2 \mathrm{D}$ ferromagnetic $\mathrm{CrI}_{3}$, including layer, polarization and temperature dependence of $A_{g}$ and $E_{g}$ modes. The non-encapsulated 2-5 layer and bulk $\mathrm{CrI}_{3}$ are rhombohedral stacking order at $10 \mathrm{~K}$, rather than monoclinic structure. The spin-phonon coupling occurs below $\sim 60 \mathrm{~K}$, resulting in the deviation of linewidth. Zero-momentum spin wave features close to the $A_{g}$ mode in frequency have been observed [34]. This work highlights the potential to manipulate spin waves through spin-phonon and magnetoelectric coupling in new ferromagnetic $2 \mathrm{D}$ materials to produce novel spintronic devices [35].

Received 31 October 2019; accepted 1 November 2019; published online 14 November 2019

1 Huang B, Clark G, Navarro-Moratalla E, et al. Layer-dependent ferromagnetism in a van der Waals crystal down to the monolayer limit. Nature, 2017, 546: 270-273

2 Gong C, Li L, Li Z, et al. Discovery of intrinsic ferromagnetism in two-dimensional van der Waals crystals. Nature, 2017, 546: 265269

3 Zhong D, Seyler KL, Linpeng X, et al. van der Waals engineering of ferromagnetic semiconductor heterostructures for spin and valleytronics. Sci Adv, 2017, 3: e1603113

4 Deng Y, Yu Y, Song Y, et al. Gate-tunable room-temperature ferromagnetism in two-dimensional $\mathrm{Fe}_{3} \mathrm{GeTe}_{2}$. Nature, 2018, 563: 94-99

5 Mounet N, Gibertini M, Schwaller P, et al. Two-dimensional materials from high-throughput computational exfoliation of experimentally known compounds. Nat Nanotech, 2018, 13: 246-252

6 Lee JU, Lee S, Ryoo JH, et al. Ising-type magnetic ordering in atomically thin FePS $_{3}$. Nano Lett, 2016, 16: 7433-7438

7 Bonilla M, Kolekar S, Ma Y, et al. Strong room-temperature ferromagnetism in $\mathrm{VSe}_{2}$ monolayers on van der Waals substrates. Nat Nanotech, 2018, 13: 289-293

8 Song T, Cai X, Tu MWY, et al. Giant tunneling magnetoresistance in spin-filter van der Waals heterostructures. Science, 2018, 360: 1214-1218

9 Klein DR, MacNeill D, Lado JL, et al. Probing magnetism in 2D van der Waals crystalline insulators via electron tunneling. Science, 2018, 360: 1218-1222

10 Wang Z, Gutiérrez-Lezama I, Ubrig N, et al. Very large tunneling magnetoresistance in layered magnetic semiconductor $\mathrm{CrI}_{3}$. Nat Commun, 2018, 9: 2516

11 Huang B, Clark G, Klein DR, et al. Electrical control of 2D magnetism in bilayer $\mathrm{CrI}_{3}$. Nat Nanotech, 2018, 13: 544-548

12 Jiang S, Shan J, Mak KF. Electric-field switching of two-dimensional van der Waals magnets. Nat Mater, 2018, 17: 406-410

13 Jiang S, Li L, Wang Z, et al. Controlling magnetism in $2 \mathrm{D} \mathrm{CrI}_{3}$ by electrostatic doping. Nat Nanotech, 2018, 13: 549-553

14 Wang Z, Zhang T, Ding M, et al. Electric-field control of magnetism in a few-layered van der Waals ferromagnetic semiconductor. Nat Nanotech, 2018, 13: 554-559

$15 \mathrm{Li} \mathrm{T}$, Jiang S, Sivadas N, et al. Pressure-controlled interlayer magnetism in atomically thin $\mathrm{CrI}_{3}$. Nat Mater, 2019, 18: 1303-1308

16 Song T, Fei Z, Yankowitz M, et al. Switching 2D magnetic states 
via pressure tuning of layer stacking. Nat Mater, 2019, 18: 12981302

17 McGuire MA, Dixit H, Cooper VR, et al. Coupling of crystal structure and magnetism in the layered, ferromagnetic insulator $\mathrm{CrI}_{3}$. Chem Mater, 2015, 27: 612-620

18 Djurdjić-Mijin S, Šolajić A, Pešić J, et al. Lattice dynamics and phase transition in $\mathrm{CrI}_{3}$ single crystals. Phys Rev B, 2018, 98: 104307

19 Sun Z, Yi Y, Song T, et al. Giant nonreciprocal second-harmonic generation from antiferromagnetic bilayer $\mathrm{CrI}_{3}$. Nature, 2019, 572: 497-501

20 Klein DR, MacNeill D, Song Q, et al. Enhancement of interlayer exchange in an ultrathin two-dimensional magnet. Nat Phys, 2019, 15: $1255-1260$

21 Sivadas N, Okamoto S, Xu X, et al. Stacking-dependent magnetism in bilayer $\mathrm{CrI}_{3}$. Nano Lett, 2018, 18: 7658-7664

22 Webster L, Liang L, Yan JA. Distinct spin-lattice and spin-phonon interactions in monolayer magnetic $\mathrm{CrI}_{3}$. Phys Chem Chem Phys, 2018, 20: 23546-23555

23 Sun L, Zheng J. Optical visualization of $\mathrm{MoS}_{2}$ grain boundaries by gold deposition. Sci China Mater, 2018, 61: 1154-1158

24 Wu S, Shi X, Liu Y, et al. The influence of two-dimensional organic adlayer thickness on the ultralow frequency Raman spectra of transition metal dichalcogenide nanosheets. Sci China Mater, 2019, 62: $181-193$

25 Zhao W, Ghorannevis Z, Amara KK, et al. Lattice dynamics in mono- and few-layer sheets of $\mathrm{WS}_{2}$ and $\mathrm{WSe}_{2}$. Nanoscale, 2013, 5: 9677-9683

26 Zhang X, Qiao XF, Shi W, et al. Phonon and raman scattering of two-dimensional transition metal dichalcogenides from monolayer, multilayer to bulk material. Chem Soc Rev, 2015, 44: 27572785

27 Xiao Y, Zhou M, Liu J, et al. Phase engineering of two-dimensional transition metal dichalcogenides. Sci China Mater, 2019, 62: 759775

28 Ren J, Teng C, Cai Z, et al. Controlled one step thinning and doping of two-dimensional transition metal dichalcogenides. Sci China Mater, 2019, 62: 1837-1845

29 Lu X, Luo X, Zhang J, et al. Lattice vibrations and Raman scattering in two-dimensional layered materials beyond graphene. Nano Res, 2016, 9: 3559-3597

30 Zhao Y, Luo X, Li H, et al. Interlayer breathing and shear modes in few-trilayer $\mathrm{MoS}_{2}$ and WSe 2 . Nano Lett, 2013, 13: 1007-1015

31 Loudon R. The Raman effect in crystals. Adv Phys, 1964, 13: 423482

32 Baum A, Milosavljević A, Lazarević N, et al. Phonon anomalies in FeS. Phys Rev B, 2018, 97: 054306

33 Granado E, García A, Sanjurjo JA, et al. Magnetic ordering effects in the Raman spectra of $\mathrm{La}_{1-x} \mathrm{Mn}_{1-x} \mathrm{O}_{3}$. Phys Rev B, 1999, 60: $11879-11882$

34 Jin W, Kim HH, Ye Z, et al. Raman fingerprint of two terahertz spin wave branches in a two-dimensional honeycomb Ising ferromagnet. Nat Commun, 2018, 9: 5122

35 Yao X, Ma J, Lin Y, et al. Magnetoelectric coupling across the interface of multiferroic nanocomposites. Sci China Mater, 2015, 58: $143-155$

Acknowledgements We acknowledge the financial support from the National Natural Science Foundation of China (51602040 and 51872039), the Science and Technology Program of Sichuan
(M112018JY0025) and the Scientific Research Foundation for New Teachers of UESTC (A03013023601007).

Author contributions Peng B developed the concept, designed the experiment and prepared the manuscript. Cheng $\mathrm{Y}$ synthesized the $\mathrm{CrI}_{3}$ crystal. Deng B, Guo K, Liu Z, Gao C and Shi Z prepared the $\mathrm{CrI}_{3}$ samples and performed the Raman measurements. Bi L, Zhou P, Zhang $\mathrm{L}, \mathrm{Lu} \mathrm{H}$ and Zhang $\mathrm{L}$ contributed to mechanism of Raman scattering.

Conflict of interest The authors declare no competing financial interests.

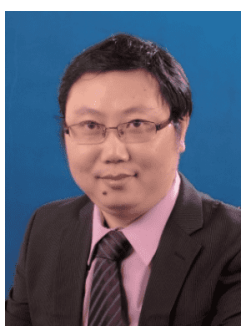

Bo Peng received his BSc (Honors) from Lanzhou University in 2005, and obtained his $\mathrm{PhD}$ degree from the Technical Institute of Physics and Chemistry, Chinese Academy of Sciences in 2010. He did his postdoctoral research in Singapore between 2010 and 2015. He is currently the Head of the Magneto-optical 2D Materials Group in the University of Electronic Science and Technology of China. His research is focused on the $2 \mathrm{D}$ ferromagnetic materials toward spintronics and valleytronics.

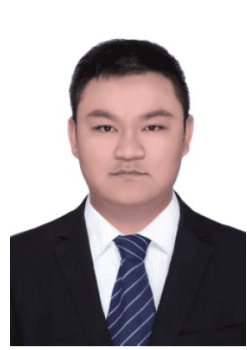

Kai Guo received his BSc from the University of Electronic Science and Technology of China in 2017. He focuses his research on the Raman studies of 2D ferromagnetic $\mathrm{CrI}_{3}$.

\section{本征铁磁二维材料 $\mathrm{CrI}_{3}$ 层间堆叠结构层数依赖性 研究}

郭锴 ${ }^{1,4}$, 邓博文 ${ }^{3}$, 刘镇 ${ }^{1,4}$, 高超峰 ${ }^{2}$, 石钟太 ${ }^{1,4}$, 毕䂞 ${ }^{1,4}$, 张丽 ${ }^{1,4}$, 陆海鹏 ${ }^{1,4}$, 周佩珩 ${ }^{1,4}$, 张林博 ${ }^{1,4^{*}}$, 程迎春 ${ }^{2}$, 彭波 ${ }^{1,4^{*}}$

摘要 二维体系中的长程铁磁序现象再次打破了Mermin-Wagner 理论. 铁磁二维材料的铁磁性与层层堆叠的顺序、结构、层间距 息息相关. 目前对铁磁二维材料 $\mathrm{CrI}_{3}$ 在低温下的堆叠顺序和结构仍 不确定, 且未见报道. 针对该科学问题, 本工作深入研究了 2-5层及 块体 $\mathrm{CrI}_{3}$ 的拉曼特征, 详细研究了拉曼特征峰与层数、偏振、旋光 和温度之间的依赖关系; 揭示了 $2-5$ 层及块体 $\mathrm{CrI}_{3}$ 在低温下 $(10 \mathrm{~K})$ 为 菱方堆叠结构, 解决了领域内关于 $\mathrm{CrI}_{3}$ 低温结构的争议, 填补了该 领域的研究空白, 并发现了自旋声子耦合现象. 本工作开创了独特 的磁光电原位传输测量系统, 从样品制备到表征完全与空气隔绝, 避免样品污染和损坏, 因此, 本工作更准确地表征出了 $\mathrm{CrI}_{3}$ 最本征 的结构特性. 